\title{
Geometric proofs of numerical stability for delay equations
}

\author{
NiCOLA Guglielmi ${ }^{\dagger}$ \\ Département di Matematica Pura e Applicata, Università dell'Aquila, via Vetoio \\ (Coppito), I-67010, L'Aquila, Italy \\ AND \\ ERNST HAIRER \\ Departimento de Mathématiques, Université de Genève, CH-1211 Genève 24, \\ Switzerland
}

[Received 10 May 1999 and in revised form 20 January 2000]

\begin{abstract}
In this paper, asymptotic stability properties of implicit Runge-Kutta methods for delay differential equations are considered with respect to the test equation $y^{\prime}(t)=a y(t)+$ $b y(t-1)$ with $a, b \in \mathbb{C}$.

In particular, we prove that symmetric methods and all methods of even order cannot be unconditionally stable with respect to the considered test equation, while many of them are stable on problems where $a \in \mathbb{R}$ and $b \in \mathbb{C}$. Furthermore, we prove that Radau-IIA methods are stable for the subclass of equations where $a=\alpha+\mathrm{i} \gamma$ with $\alpha, \gamma \in \mathbb{R}, \gamma$ sufficiently small, and $b \in \mathbb{C}$.
\end{abstract}

\section{Introduction}

In this work we study the asymptotic stability behavior of numerical methods when applied to the test equation

$$
y^{\prime}(t)=a y(t)+b y(t-1), \quad t>0,
$$

with initial condition $y(t)=g(t),-1 \leqslant t \leqslant 0$, where the constants $a, b$ and the function $g(t)$ are all complex. We are mainly interested in methods that produce unconditionally stable numerical solutions for all $a$ and $b$, for which the exact solution tends to zero.

Most of the publications on this subject restrict their analysis to the cone $|b|<-\Re a$, where $A$-stability has been proven to be a necessary and sufficient condition for preserving the asymptotic stability of the solution. Concerning the purely retarded equation, that is (1.1) with $a=0$, this kind of topic has first been investigated by Cryer (1974) and van der Houwen \& Sommeijer (1984), who have considered linear multistep methods. For the case of real $a$ and $b$, a stability analysis has recently been given by Guglielmi (1997) and Guglielmi \& Hairer (1999). There, some numerical experiments on the complex coefficient test equation indicated that Gauss methods are not stable, while Radau-IIA methods could be stable. Recently, Maset (2000) proved the stability of the backward Euler method for (1.1) with complex coefficients.

\footnotetext{
†Email: guglielm@univaq.it

†Email: Ernst.Hairer@math.unige.ch
} 
The paper is organized as follows. In Section 2 we give the stability definitions and introduce the root locus curves, which are essential for the technique used in this work. In Section 3 we give an instability result for the class of symmetric methods. In the subsequent section (Section 4), we give stability results for both symmetric and nonsymmetric methods when restricted to equations with $a \in \mathbb{R}$ but allowing $b \in \mathbb{C}$. Finally, in Section 5 , we show by a local perturbation analysis that no method with even order can be stable for the general complex coefficient test equation (1.1). For methods with odd order, we discuss the stability for values of $a$ close to the real axis.

We consider Runge-Kutta methods applied with constant stepsize $h=1 / m$, and we use the internal stage value $g_{i}$ of a previous step as an approximation to $y\left(t_{n}+c_{i} h-1\right)$. For the test equation (1.1) this yields the recursion

$$
\begin{aligned}
& g_{i}^{(n)}=y_{n}+h \sum_{j=1}^{s} a_{i j}\left(a g_{j}^{(n)}+b g_{j}^{(n-m)}\right) \\
& y_{n+1}=y_{n}+h \sum_{i=1}^{s} b_{i}\left(a g_{i}^{(n)}+b g_{i}^{(n-m)}\right),
\end{aligned}
$$

where $s$ is the number of stages and $b_{i}, c_{i}, a_{i j}$ are the usual coefficients of the Runge-Kutta method (see Zennaro, 1986).

\section{Stability regions}

It is difficult to represent geometrically the set of all $(a, b) \in \mathbb{C} \times \mathbb{C}$ such that 1.1) is stable. Following Guglielmi (1998) we therefore fix $a \in \mathbb{C}$, and we consider the set of all $b \in \mathbb{C}$ such that stability occurs.

\subsection{Analytical stability region}

For a fixed $a \in \mathbb{C}$ we denote by $Q_{\star}[a]$ the set of complex numbers $b$ such that the solution of (1.1) satisfies $\lim _{t \rightarrow \infty} y(t)=0$ for all initial functions $g(t)$. By looking at solutions of the form $y(t)=\mathrm{e}^{\lambda t}$ we are led to the characteristic equation

$$
\mathcal{K}(\lambda ; a, b):=\lambda-a-b \mathrm{e}^{-\lambda}=0 .
$$

It is known (see e.g., El'sgol'ts \& Norkin, 1973) that this set is given by

$$
Q_{\star}[a]=\{b \in \mathbb{C} ; \text { all roots of (2.1) satisfy } \Re \lambda<0\} .
$$

Figure 1 shows $Q_{\star}[a]$ (white region) for $a=0.2$ (left picture) and for $a=-3$ (right picture). For real $a$, the set $Q_{\star}[a]$ has the form of a lying drop. It satisfies $Q_{\star}\left[a_{1}\right] \subset Q_{\star}\left[a_{2}\right]$ whenever $a_{1} \geqslant a_{2}$, and the intersections with the horizontal axis are at $b=-a$ and $b=-\phi_{a} / \sin \phi_{a}$ where $\phi_{a} \in(0, \pi)$ is defined by $a=\phi_{a} \cot \phi_{a}$. This is a consequence of the shape of the stability region for the real case. It is interesting to note that the size of the stability region $Q_{\star}[a]$ only depends on the real part of $a$. Indeed, we have for $\gamma \in \mathbb{R}$ that

$$
Q_{\star}[a+\mathrm{i} \gamma]=\mathrm{e}^{\mathrm{i} \gamma} Q_{\star}[a],
$$



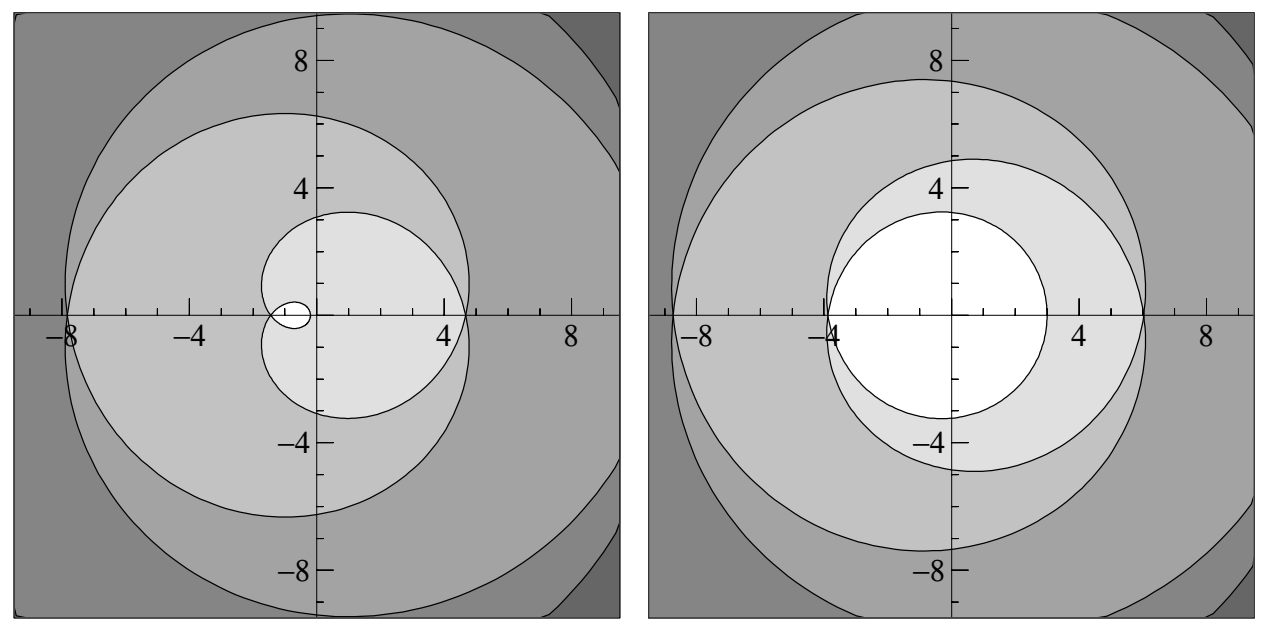

FIG. 1. The stability set $Q_{\star}[a]$ (white region) for $a=0 \cdot 2$ (left) and $a=-3$ (right). The intensity of grey indicates the increasing number of roots of (2.1) lying in the right half plane.

so that $Q_{\star}[a+\mathrm{i} \gamma]$ is obtained from $Q_{\star}[a]$ by a simple rotation around the origin. This follows from the fact that $\mathcal{K}(\lambda ; a+\mathrm{i} \gamma, b)=\mathcal{K}\left(\lambda-\mathrm{i} \gamma ; a, b \mathrm{e}^{-\mathrm{i} \gamma}\right)$.

In Fig. 1 we have also drawn the curve that corresponds to the set of $b$ where at least one root of (2.1) lies on the imaginary axis. It is given by

$$
b_{\star}(\phi)=\mathrm{e}^{\mathrm{i} \phi}(\mathrm{i} \phi-a), \quad \phi \in \mathbb{R},
$$

and is commonly called the root locus curve (see e.g., Baker \& Paul, 1994). Since the roots of (2.1) depend continuously on $b$, the curve (2.3) separates regions with different numbers of roots lying in the right half plane.

\subsection{Numerical stability region}

For fixed $a \in \mathbb{C}$ and $m \in \mathbb{Z}^{+}$, we denote by $Q_{m}[a]$ the set of complex numbers $b$ such that the numerical solution $\left\{y_{n}\right\}_{n} \geqslant 0$ of (1.2), (1.3) with constant stepsize $h=1 / m$, satisfies $\lim _{n \rightarrow \infty} y_{n}=0$ for all initial functions $g(t)$. If we look at numerical approximations of the form $y_{n}=\zeta^{n} y_{0}, g_{i}^{(n)}=\zeta^{n} g_{i}$, we are led to the characteristic equation

$$
\zeta=R(z), \quad m z=a+b \zeta^{-m},
$$

where $R(z)$ is the stability function of the method (see for example Section IV.2 of Hairer $\&$ Wanner, 1996). Similar to the analysis for the analytical solution, one can show that the set $Q_{m}[a]$ is given by

$$
Q_{m}[a]=\{b \in \mathbb{C} ; \text { all roots of (2.4) satisfy }|\zeta|<1\} .
$$

DEFINITION 2.1 A numerical one-step method with stability function $R(z)$ is called $\tau$ stable if

$$
Q_{\star}[a] \subseteq \bigcap_{m=1}^{\infty} Q_{m}[a] \quad \text { for all } a \in \mathbb{C}
$$



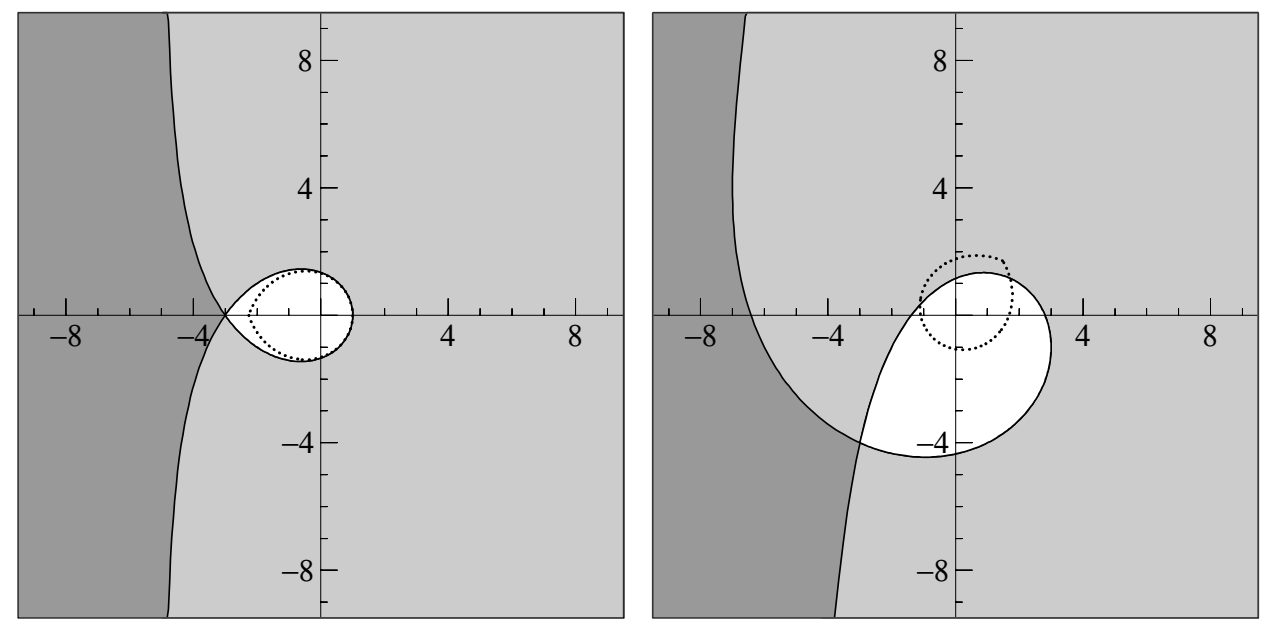

FIG. 2. The numerical stability set $Q_{m}[a]$ with $m=1$ (white region) for the trapezoidal rule, for $a=-1$ (left) and $a=-1+4 \mathrm{i}$ (right). The dotted curve indicates the exact stability domain $Q_{\star}[a]$.
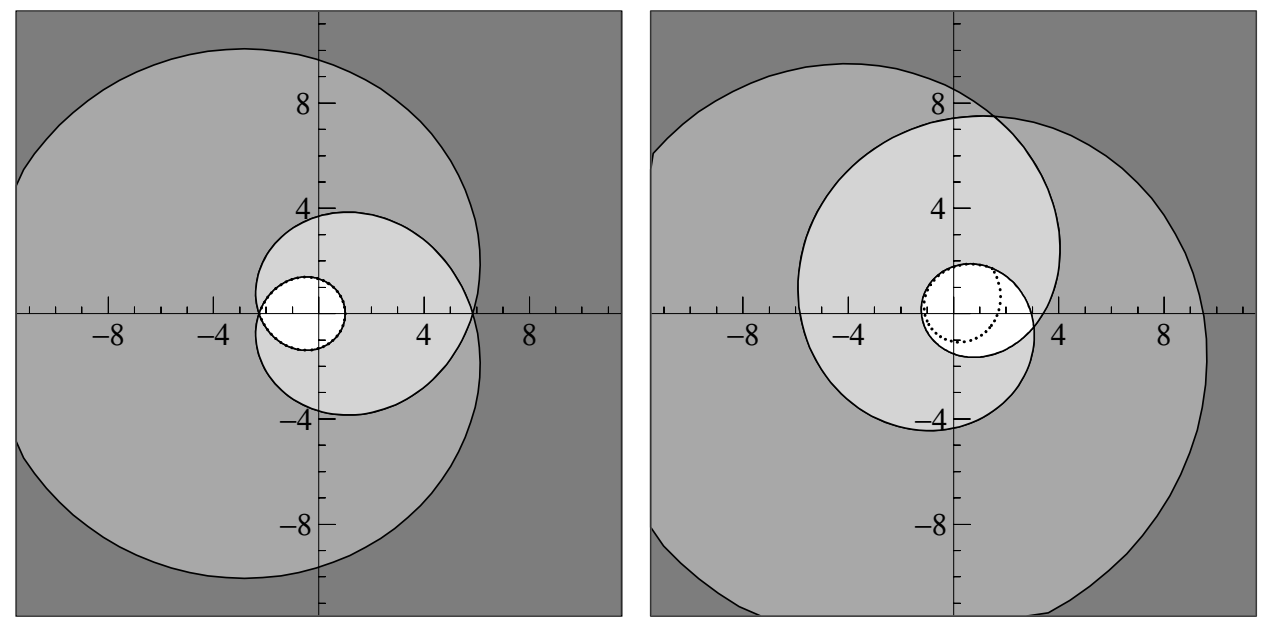

FIG. 3. The numerical stability set $Q_{m}[a]$ with $m=1$ (white region) for the subdiagonal Padé approximation $R_{2,3}(z)$, for $a=-1$ (left) and $a=-1+4 \mathrm{i}$ (right). The dotted curve indicates the exact stability domain $Q_{\star}[a]$.

In order to plot the region $Q_{m}[a]$ we have to parametrize the set of $z$ satisfying $|R(z)|=$ 1. For this purpose we recall a result from Guglielmi \& Hairer (1999), which is fundamental for the stability analysis of later sections.

LEMMA 2.2 Suppose that the whole boundary of the stability domain $S=\{z ;|R(z)| \leqslant$ $1\}$ can be described by one smooth injective curve $z(t)=x(t)+\mathrm{i} y(t)$ for $t \in(-c, c)$, which satisfies $z(-t)=\bar{z}(t)$ and $z(0)=0$, and which is oriented in such a way that $S$ lies 
to its left. We allow $c=+\infty$ and we assume that $\lim _{t \rightarrow c} z(t)$ is either $\infty$ (on the Riemann sphere) or on the real axis. We further define the smooth function $\varphi(t)$ by $\varphi(0)=0$ and

$$
R(z(t))=\mathrm{e}^{\mathrm{i} \varphi(t)}, \quad \text { i.e., } \quad \varphi(t)=-\mathrm{i} \log R(z(t)) .
$$

Then, the function $\varphi(t)$ is strictly monotonically increasing and satisfies $\varphi(-t)=-\varphi(t)$ and $\lim _{t \rightarrow c} \varphi(t)=s \pi$, where $s$ is the number of poles of $R(z)$.

The set of values $b$, for which at least one of the roots $\zeta$ of (2.4) satisfies $|\zeta|=1$, is given by

$$
b_{m}(t)=\mathrm{e}^{\mathrm{i} m \varphi(t)}(\mathrm{i} m y(t)+m x(t)-a),
$$

where $x(t), y(t)$, and $\varphi(t)$ are as in Lemma 2.2. We call this the (numerical) root locus curve.

EXAMPLE 2.3 (Trapezoidal rule) Figure 2 shows the curve $b_{m}(t)$ for $m=1$ and for the trapezoidal rule

$$
R(z)=\frac{1+z / 2}{1-z / 2} .
$$

This curve divides the complex plane into three domains, containing 0,1 , and 2 roots $\zeta$ of (2.4) outside the unit disc. The reason for the finite number of subdomains (which is in contrast to the situation of Fig. 1) is the following: if the stability function is given by $R(z)=P(z) / Q(z)$ with polynomials $P(z)$ and $Q(z)$, then (2.4) is equivalent to

$$
\zeta=R(z), \quad P(z)^{m}(m z-a)=b Q(z)^{m},
$$

which is a polynomial in $z$ and therefore has only finitely many solutions. In the case of the trapezoidal rule and $m=1,(2.8)$ is a polynomial of degree 2. Comparison with the analytical stability region shows that $Q_{m}[a] \supseteq Q_{\star}[a]$ for the real value $a=-1$, but $Q_{m}[a] \nsupseteq Q_{\star}[a]$ for $a=-1+4$ i. Hence, the trapezoidal rule is not $\tau$-stable (Guglielmi, 1998).

EXAMPLE 2.4 (Padé approximation) We repeat the experiment of the previous example with the subdiagonal Padé approximation

$$
R_{2,3}(z)=\frac{1+2 z / 5+z^{2} / 20}{1-3 z / 5+3 z^{2} / 20-z^{3} / 60} .
$$

Since $R_{2,3}(\infty)=0$, the root locus curve (2.7) is bounded (Fig. 3). This time we observe that for both values of $a$ the analytical stability region is completely contained in the numerical stability region. Due to the high order (order 5 for (2.9) in contrast to order 2 for the trapezoidal rule) the numerical stability region much better approximates the set $Q_{\star}[a]$.

\section{Instability results}

We start our analysis by considering $A$-stable symmetric Runge-Kutta methods. Although most of them are stable for (1.1) when $a$ and $b$ are real, we shall see in this section that none of them can be $\tau$-stable. 
Recall that a Runge-Kutta method is symmetric if it coincides with its adjoint, i.e., with its inverse applied with stepsize $-h$ (see e.g., Section II.8 of Hairer et al., 1993). The stability function of a symmetric method therefore satisfies $R(-z) R(z) \equiv 1$. If it is $A$-stable, the stability domain $S$ is exactly the negative half-plane and the boundary $\partial S$ can conveniently be parametrized by $y$. Since $x=0$ for values on $\partial S$, we get

$$
b_{m}(y)=\mathrm{e}^{\mathrm{i} m \varphi(y)}(\mathrm{i} m y-a), \quad y \in \mathbb{R},
$$

where the function $\varphi(y)$ is defined by $R(\mathrm{i} y)=\mathrm{e}^{\mathrm{i} \varphi(y)}$ (see Lemma 2.2). The properties of $\varphi(y)$ and of its inverse $y(\varphi)$ are discussed in Guglielmi \& Hairer (1999). Note also that the only difference in formulæ (3.1) and (2.3) (when we set $\phi=m y$ ) is in the function $\varphi(y)$, which in fact is an approximation to $y$.

THEOREM 3.1 Symmetric Runge-Kutta methods cannot be $\tau$-stable.

Proof. For $b=0$, equation (1.1) becomes the well-known Dahlquist test equation for $A$-stability. Hence, $A$-stability is a necessary condition for $\tau$-stability, and the numerical root locus curve can be assumed to be given by (3.1). The idea of the proof is to look at the point $b_{0}$ of this curve, which has minimal distance to the origin. Since $\left|b_{m}(y)\right|^{2}=$ $\Re a^{2}+(m y-\Im a)^{2}$, it is obtained for $y=\Im a / m$.

We now let $a \in \mathbb{C}$ be such that $\Re a<0$, and $y=\Im a / m$ satisfies $m \varphi(y) \neq m y+2 k \pi$. The assumption $\Re a<0$ implies that $b_{0} \in \partial Q_{m}[a]$, because in this case $0 \in Q_{m}[a]$, and the segment joining 0 and $b_{0}$ does not intersect the root locus curve. The assumption on $\Im a$ implies that $b_{0}$ does not lie on the analytical root locus curve. Since the minimal distance of the analytical root locus curve to the origin is also $|\Re a|$, this implies that $b_{0}$ is in the interior of $Q_{\star}[a]$. Consequently, the necessary condition for $\tau$-stability, $Q_{m}[a] \supseteq Q_{\star}[a]$, cannot hold.

Gauss methods are $s$-stage Runge-Kutta methods of optimal order $2 s$ (see Section IV.5 of Hairer \& Wanner, 1996). Since they are symmetric we have the following result.

COROLlary 3.2 None of the Gauss methods is $\tau$-stable.

This extends a result of Guglielmi (1998), which states that the trapezoidal rule (and the implicit mid-point rule) is not $\tau$-stable (see Fig. 2).

The above proof also shows that the condition $Q_{m}[a] \supseteq Q_{\star}[a]$ is violated by symmetric Runge-Kutta methods for nearly all $a \in \mathbb{C}$. An exceptional case will be treated in the following section.

\section{Stability results}

In this section we consider the case where $a \in \mathbb{R}$, but $b \in \mathbb{C}$. Here, the analytical and numerical stability regions are symmetric with respect to the real axis (see Figs 1, 2, and 3). Similar to the case where $a \in \mathbb{R}$ and $b \in \mathbb{R}$ (Guglielmi \& Hairer, 1999) we shall derive positive stability results for symmetric as well as for non-symmetric methods.

We mention that the test equation considered in this section is a generalization of the purely retarded test equation (that is when $a=0$ ). The stability of numerical methods for the purely retarded test equation has been previously studied by Cryer (1974), 
Barwell (1975), Al Mutib (1984) and van der Houwen \& Sommeijer (1984). In particular, Barwell called the numerical method $Q$-stable if the numerical stability region contains the analytical stability region. In our notation this means that $Q_{m}[0] \supseteq Q_{\star}[0]$ for all $m \in \mathbb{Z}^{+}$.

\subsection{Symmetric methods}

Our analysis for symmetric methods is based on Lemma 2 of Guglielmi \& Hairer (1999), which we recall here.

LEMMA 4.1 Let $R(z)$ be symmetric, so that the order star

$$
A=\left\{z \in \mathbb{C} ;|R(z)|>\left|\mathrm{e}^{z}\right|\right\}
$$

has the whole imaginary axis as boundary. Assume that $A$ touches the imaginary axis everywhere from the left side. Then, the function $\varphi(y)$ defined by $R(\mathrm{i} y)=\mathrm{e}^{\mathrm{i} \varphi(y)}$ and $\varphi(0)=0$ satisfies

$$
\varphi(y)<y \quad \text { for } \quad y>0 \text {. }
$$

In that paper it is also proved that Gauss methods fulfil the assumptions of Lemma 4.1. In particular, for the mid-point rule (one-stage Gauss method) we have $\varphi(y)=$ $2 \arctan (y / 2)$.

THEOREM 4.2 If $a$ is real, and if the symmetric stability function $R(z)$ satisfies the assumptions of Lemma 4.1, then

$$
Q_{m}[a] \supset Q_{\star}[a] \quad \text { for all } m \geqslant 1
$$

Proof. Due to the symmetry of the stability regions with respect to the real axis, it is sufficient to consider the upper half plane $\Im b>0$. We use

$$
b_{m}(y)=\mathrm{e}^{\mathrm{i} m(\varphi(y)-y)} b_{\star}(m y),
$$

which follows immediately from (3.1) and (2.3). We shall show that the whole root locus curve $b_{m}(y)$ (with the exception of the point $b=-a$ ) lies outside of $Q_{\star}[a]$. This is obvious for $m y \geqslant \pi$, because $\left|b_{m}(y)\right|^{2}=\left|b_{\star}(m y)\right|^{2}=(m y)^{2}+a^{2}$ is monotonically increasing, and the maximal distance of $Q_{\star}[a]$ from the origin is $\left|b_{\star}\left(\phi_{a}\right)\right|$ with $\phi_{a} \in(0, \pi)$ given by $a=\phi_{a} \cot \phi_{a}$ (Fig. 1). For $0<m y<\pi$, this follows from $0<\varphi(y)<y$ (Lemma 4.1) and from (4.2), because the factor $\mathrm{e}^{\mathrm{i} m(\varphi(y)-y)}$ represents a clockwise rotation with an angle of less than $m y$ degrees. Since the intersection of $Q_{\star}[a]$ with the circle $\left\{b ;|b|=\left|b_{\star}(m y)\right|\right\}$ is a connected arc containing the left intersection point with the real axis, this implies that $b_{m}(y)$ lies outside $Q_{\star}[a]$ also in this case. The statement of the theorem now follows from the fact that all $b \in Q_{\star}[a] \cap \mathbb{R}$ lie in $Q_{m}[a] \cap \mathbb{R}$ (Theorem 2 of Guglielmi \& Hairer, 1999).

REMARK 4.3 An interesting consequence of the previous result is that Gauss methods are stable when $a$ is real and in particular, according to the definition given by Barwell (1975), they are $Q$-stable. 

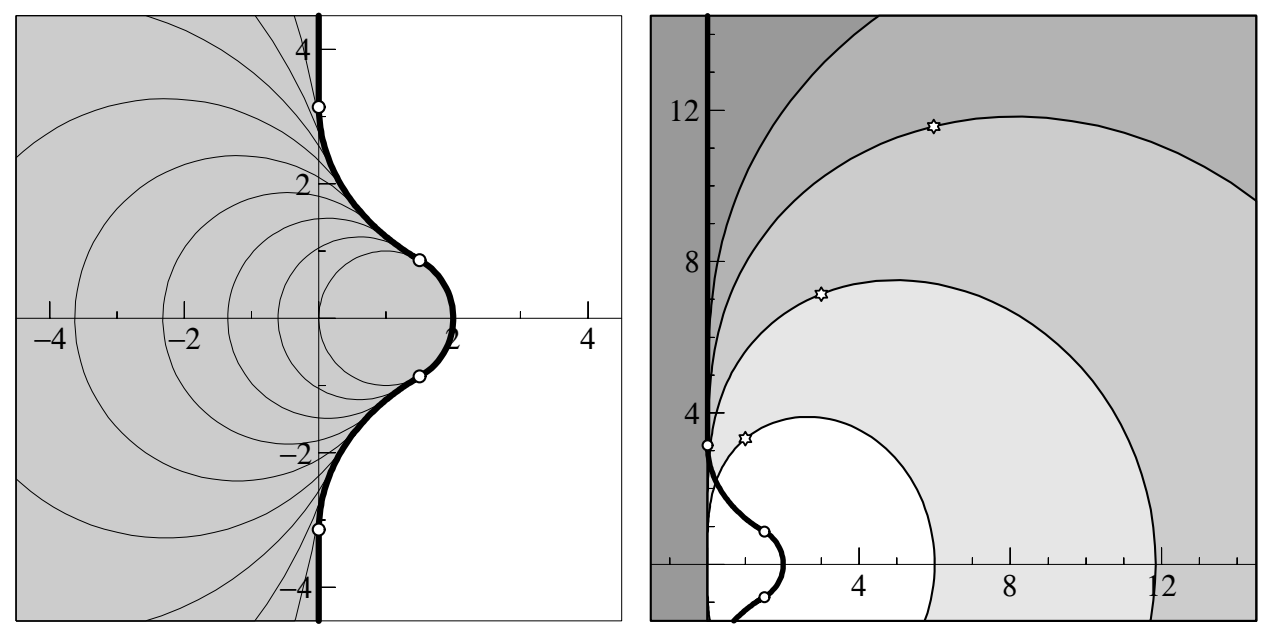

FIG. 4. The left-hand picture shows the discs $D[a]$ of (4.3) and their union $D$. The picture on the right shows the stablity domains of the Radau IIA methods with $s=2,3,4,5$ stages together with the boundary of $D$. By Table 1 of Guglielmi \& Hairer (1999), condition (i) of Theorem 4.4 is satisfied for all values between the origin and the point marked by an asterix.

\subsection{Non-symmetric methods}

We next consider arbitrary non-symmetric stability functions, and we give sufficient conditions for numerical stability. Similar to the proof of the previous theorem, we study conditions under which the numerical root locus curve does not enter the analytical stability region. For a fixed $a \leqslant 1$, the point $b_{m}(t)$ of (2.7) is certainly outside of $Q_{\star}[a]$ if its distance to the origin is larger than $b_{\star}\left(\phi_{a}\right)$ with $\phi_{a}$ as in the proof of Theorem 4.2. Because $\left|b_{m}(t)\right|=|m z(t)-a|$, this is the case if $m z(t) \notin D[a]$, where

$$
D[a]=\left\{z \in \mathbb{C} ;|z-a|<\left|b_{\star}\left(\phi_{a}\right)\right|\right\} .
$$

The set $D[a]$ for several values of $a$, and the union

$$
D=\bigcup_{a \leqslant 1} D[a]
$$

are shown in the left-hand picture of Fig. 4. Computing the envelope of this family of circles, one finds that the set $D$ lies to the left of the curve given by the imaginary axis for $|y| \geqslant \pi$, by

$$
x(\phi)=\frac{\phi \sin ^{2} \phi}{\phi-\sin \phi \cos \phi}, \quad y(\phi)= \pm \frac{\phi \sqrt{\phi^{2}-\sin ^{2} \phi}}{\phi-\sin \phi \cos \phi} \quad \text { for } \quad \phi \in(0, \pi)
$$

and by the circle with radius 1 and centre 1 between the points $(3 / 2, \pm \sqrt{3} / 2)$.

THEOREM 4.4 Let $a$ be real. In the situation of Lemma 2.2, assume that $Q_{m}[a] \cap Q_{\star}[a] \neq$ $\emptyset$ for all $m$, and that for every $t$ at least one of the following two conditions is satisfied: 

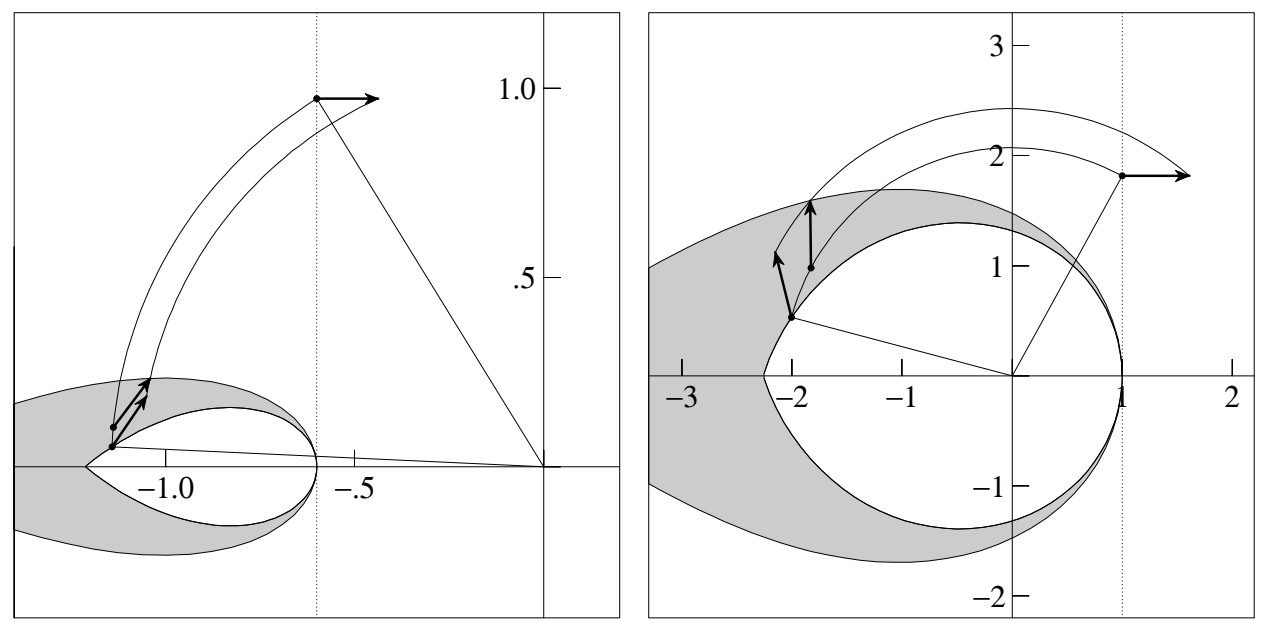

FIG. 5. Illustration of the proof of Theorem 4.4 with the $\theta$-method $R(z)=(1+(1-\theta) z) /(1-\theta z)$ and $\theta=2 / 3$ (left, $a=0 \cdot 6$; right, $a=-1$ ). The grey region is the set $Q_{m}[a]$ minus $Q_{\star}[a]$.

(i) $x(t) \geqslant 0$ and $y(t) \geqslant \varphi(t)$

(ii) $z(t)=x(t)+\mathrm{i} y(t) \notin D$.

Then, it holds that $Q_{m}[a] \supset Q_{\star}[a]$ for all $m \geqslant 1$.

Proof. Because of $Q_{m}[a] \cap Q_{\star}[a] \neq \emptyset$, it is sufficient to prove that the numerical root locus curve $b_{m}(t)$ never enters the analytical stability region $Q_{\star}[a]$. If $m z(t) \notin D$, which is the case for $|m y(t)| \geqslant \pi$ or when condition (ii) is satisfied, this is true by the definition of $D$. We therefore have to consider only the case where $x(t) \geqslant 0$ and $0 \leqslant m \varphi(t) \leqslant m y(t)<\pi$.

The idea is to write the root locus curve (2.7) as

$$
b_{m}(t)=\mathrm{e}^{\mathrm{i} m \varphi(t)}\left(\mathrm{e}^{-\mathrm{i} m y(t)} b_{\star}(m y(t))+m x(t)\right),
$$

and to interpret this equation geometrically as follows (Fig. 5): we start by considering the point $b_{\star}(m y(t))$ on the boundary of the analytical stability domain $Q_{\star}[a]$, we multiply it by $\mathrm{e}^{-\mathrm{i} m y(t)}$ (this is a rotation around the origin and yields the point imy $(t)-a$ on the vertical line $\mathfrak{R} b=-a)$, then we add the positive number $m x(t)$, and finally we multiply by $\mathrm{e}^{\mathrm{i} m \varphi(t)}$ (a rotation back). In order to prove that $b_{m}(t)$ lies outside $Q_{\star}[a]$, we consider the horizontal arrow from the point imy $(t)-a$ to imy $(t)-a+m x(t)$ (Fig. 5). A multiplication by $\mathrm{e}^{\mathrm{i} m y(t)}$ would turn it back with one end of the arrow at $b_{\star}(m y(t))$ and the other end outside of the analytic stability region. This is because the tangent vector of the analytical root locus curve at $b_{\star}(\phi)$ with $\phi=m y(t)$ is given by $b_{\star}^{\prime}(\phi)=\mathrm{i} \mathrm{e}^{\mathrm{i} \phi}(1-a+\mathrm{i} \phi)$, the exterior normal vector is given by $u=\mathrm{e}^{\mathrm{i} \phi}(1-a+\mathrm{i} \phi)$, and the inner product of $u$ with the vector $v=\mathrm{e}^{\mathrm{i} \phi} m x(t)$, which is given by $\Re(\bar{u} v)$, is positive because of $a<1$. A multiplication by $\mathrm{e}^{\mathrm{i} m \varphi(t)}$, which brings the peak of the arrow to $b_{m}(t)$ on the boundary of $Q_{m}[a]$, corresponds to a rotation by a smaller angle $(\varphi(t) \leqslant y(t))$, so that $b_{m}(t)$ also stays clearly outside $Q_{\star}[a]$.

As an interesting application of the previous theorem we consider the $s$-stage RadauIIA methods for $s \geqslant 2$. Figure 4 (right) shows the stability domains for $s=2,3,4$, and 
5, as well as the boundary of the set $D$ of (4.4). Most of the boundary of the stability domains is seen to lie outside of $D$ (condition (ii) of Theorem 4.4), so that condition (i) has to be checked only for a small part of it. In fact, condition (i) of Theorem 4.4 follows immediately from Table 1 of Guglielmi \& Hairer (1999) for $2 \leqslant s \leqslant 7$. The case $s=1$ (backward Euler method) has recently been investigated by Maset (2000), who proved its stability for $a \in \mathbb{C}$ and $b \in \mathbb{C}$.

\section{A local perturbation analysis}

We conclude our investigation with a local analysis close to $b=-a$. This point lies on the analytical as well as on the numerical root locus curve, and the two curves are there tangential to each other. Throughout this section we assume that $|a|<\left|b_{\star}\left(\phi_{a}\right)\right|$ with $\phi_{a} \in$ $(0, \pi)$ given by $\Re a=\phi_{a} \cot \phi_{a}$, so that $b=-a$ lies on the boundary of the analytical stability region $Q_{\star}[a]$. Let the stability function $R(z)$ satisfy

$$
R(z)=\mathrm{e}^{z}\left(1-C z^{p+1}+\mathcal{O}\left(z^{p+2}\right)\right)
$$

where $C \neq 0$ is the error constant. We parametrize the boundary of the stability domain $S$ in terms of the angle $\varphi$, so that $R(z(\varphi))=\mathrm{e}^{\mathrm{i} \varphi}$. Because of (5.1), we get for $\varphi \rightarrow 0$ that

$$
z(\varphi)=\mathrm{i} \varphi+C(\mathrm{i} \varphi)^{p+1}+\mathcal{O}\left(\varphi^{p+2}\right)
$$

Inserted into the root locus curve (2.7) this yields

$$
b_{m}(\varphi)-b_{\star}(m \varphi)=\mathrm{e}^{\mathrm{i} m \varphi}(m z(\varphi)-\mathrm{i} m \varphi)=m C(\mathrm{i} \varphi)^{p+1}+\mathcal{O}\left(\varphi^{p+2}\right) .
$$

We now distinguish the cases where $p$ is even or odd.

THEOREM 5.1 (Even order) One-step methods of even order cannot be $\tau$-stable.

Proof. The tangent vector of the analytical root locus curve at $b_{\star}(0)=-a$ is in the direction $b_{\star}^{\prime}(0)=(1-a) i$, which is non-vertical as soon as $\Im a \neq 0$. For even order $p=2 k$, it follows from (5.2) that

$$
b_{m}(\varphi)-b_{\star}(m \varphi)=\mathrm{i}(-1)^{k} m C \varphi^{2 k+1}+\mathcal{O}\left(\varphi^{2 k+2}\right),
$$

so that close to $b=-a$ (i.e., for positive and negative small $\varphi$ ) the numerical root locus curve lies on both sides of $b_{\star}(m \varphi)$. This contradicts $\tau$-stability.

Since the order of symmetric methods is always even, this result gives a second proof of the statement of Theorem 3.1. In Section 3 we have shown more for symmetric methods, namely that for nearly all $a$ we have $Q_{m}[a] \not \supset Q_{\star}[a]$ and not simply for $a$ satisfying $|a|<\left|b_{\star}\left(\phi_{a}\right)\right|$.

THEOREM 5.2 (Odd order) Consider a method of odd order $p=2 k-1$. For every $a \in \mathbb{C}$ there exists a neighbourhood $U$ of $b=-a$ such that $U \cap Q_{m}[a] \supset U \cap Q_{\star}[a]$ if and simply if

$$
(-1)^{k} C>0
$$


Moreover, assuming that $Q_{m}\left[a_{0}\right] \supset Q_{\star}\left[a_{0}\right]$ for a real $a_{0}<1$, and that the boundary of $Q_{m}[a]$ touches $Q_{\star}[a]$ only at the point $b=-a$, then condition (5.3) implies that $Q_{m}[a] \supset$ $Q_{\star}[a]$ for all $a$ in a complex neighbourhood of $a_{0}$.

Proof. The first statement follows from

$$
b_{m}(\varphi)-b_{\star}(m \varphi)=(-1)^{k} m C \varphi^{2 k}+\mathcal{O}\left(\varphi^{2 k+1}\right),
$$

and from the fact that close to $b_{\star}(0)=-a$ the region $Q_{\star}[a]$ lies to the left of the curve $b_{\star}(\phi)$. The second statement follows from a continuity argument.

We remark that (5.3) is also a necessary condition for $\tau(0)$-stability (see Guglielmi \& Hairer, 1999). The stability function of the Radau-IIA methods satisfies (5.3), so that the stability result of Theorem 5.2 applies.

\section{Conclusions}

In this work we have concluded the characterization for $A$-stable symmetric methods when applied to the test equation (1.1). In particular, this class of methods, which has been proved to be stable for real coefficient delay differential equations (under some further technical assumptions, as shown by Guglielmi \& Hairer, 1999), performs stably on equation (1.1) when $a$ is real and $b$ complex, but turns out to be unstable on general complex coefficient problems. Moreover, we have shown by a local perturbation analysis that no method of even order can be stable for the general complex coefficient test equation (1.1).

On the other hand we have shown that Radau-IIA methods (with the number of stages $s \leqslant 7$ ) perform stably on problems with real $a$, and also in the case where the imaginary part of $a$ is sufficiently small. A complete proof of $\tau$-stability for the Radau-IIA methods is still missing.

\section{Acknowledgement}

Nicola Guglielmi wishes to thank the Italian M.U.R.S.T. for partially supporting this work.

\section{REFERENCES}

AL MutiB, A. N. 1984 Stability properties of numerical methods for solving delay differential equations. J. Comput. Appl. Math. 10, 71-79.

BARWELL, V. K. 1975 Special stability problems for functional differential equations. BIT 15, 130135.

BAKER, C. T. H. \& PAUL, C. A. H. 1994 Computing stability regions-Runge-Kutta methods for delay differential equations. IMA J. Numer. Anal. 14, 347-362.

CrYer, C. W. 1974 Highly stable multistep methods for retarded differential equations. SIAM J. Numer. Anal. 11, 788-797.

EL'SGOL'TS, L. E. \& Norkin, S. B. 1973 Introduction to the Theory and Application of Differential Equations with Deviating Arguments. New York: Academic.

Guglielmi, N. 1997 On the asymptotic stability properties of Runge-Kutta methods for delay differential equations. Numer. Math. 77, 467-485. 
Guglielmi, N. 1998 Delay dependent stability regions of $\Theta$-methods for delay differential equations. IMA J. Numer. Anal. 18, 399-418.

Guglielmi, N. \& HaIRER, E. 1999 Order stars and stability for delay differential equations. Numer. Math. 83, 371-383.

Hairer, E., Nørsett, S. P., \& WAnner, G. 1993 Solving Ordinary Differential Equations I. Nonstiff Problems., 2nd edn. (Springer Series in Computational Mathematics) 8, Berlin: Springer.

HAIRER, E. \& WANner, G. 1996 Solving Ordinary Differential Equations II. Stiff and DifferentialAlgebraic Problems, 2nd edn. (Springer Series in Computational Mathematics) 14, Berlin: Springer.

VAn Der Houwen, P. J. \& SommeiJer, B. P. 1984 Stability in linear multistep methods for pure delay equations. J. Comput. Appl. Math. 10, 55-63.

MASET, S. 2000 Stability of Runge-Kutta methods for linear delay differential equations. Numer. Math. at press.

Zennaro, M. $1986 P$-stability properties of Runge-Kutta methods for delay differential equations. Numer. Math. 49, 305-318. 